\title{
CHARACTERIZATION OF PSEUDOCOMPACTNESS BY THE TOPOLOGY OF UNIFORM CONVERGENCE ON FUNCTION SPACES
}

\author{
R. A. MCCOY \\ (Received 18 August 1976; revised 18 February, 9 June 1977) \\ Communicated by W. D. Wallis
}

\begin{abstract}
It is shown that a Tychonoff space $X$ is pseudocompact if and only if for every metrizable space $Y$, all uniformities on $Y$ induce the same topology on the space of continuous functions from $X$ into $Y$. Also for certain pairs of spaces $X$ and $Y$, a necessary and sufficient condition is established in order that all uniformities on $Y$ induce the same topology on the space of continuous functions from $X$ into $Y$.
\end{abstract}

Subject classification (Amer. Math. Soc. (MOS) 1970): primary 54C35, secondary 54D99. Keywords: Function spaces, topology of uniform convergence, open-cover topology, pseudocompact.

Let $X$ and $Y$ be topological spaces, and let $Y^{X}$ denote the space of continuous functions from $X$ into $Y$. Every uniformity on $Y$ induces a uniformity on $Y^{X}$, which in turn generates a topology on $Y^{X}$. Two compatible uniformities on $Y$ (that is, uniformities generating the same topology) may not induce compatible uniformities on $Y^{X}$. However, if $X$ is compact, then the induced uniformities on $Y^{X}$ will always be compatible, since they will all generate the compact-open topology. In the case that $Y$ is metrizable, this compactness of $X$ can be weakened to $X$ being pseudocompact, that is, a Tychonoff space such that every real-valued continuous function on it is bounded. So that when $X$ is pseudocompact and $Y$ is metrizable, then all compatible uniformities on $Y$ induce the same topology on $Y^{x}$, though this need not be the compact-open topology.

It will be convenient to compare the topology of uniform convergence with the open-cover topology. This topology was first introduced independently by Poppe (1966) and Irudayanathan (1967), and is also discussed by McCoy (1977). The following two theorems then summarize the propositions 
in this paper. Here $R$ denotes the space of real numbers with the usual topology.

THEOREM 1. The following are equivalent for a Tychonoff space $X$.

1. $X$ is pseudocompact.

2. For every metrizable space $Y$, all compatible uniformities on $Y$ induce the open-cover topology on $Y^{X}$.

3. For every metrizable space $Y$, all compatible uniformities on $Y$ induce the same topology on $Y^{X}$.

4. All compatible uniformities on $R$ induce the open-cover topology on $R^{x}$.

5. All compatible uniformities on $R$ induce the same topology on $R^{x}$.

6. There exists a metrizable space $Y$ containing a nontrivial path such that all compatible uniformities on $Y$ induce the open-cover topology on $Y^{X}$.

7. There exists a space $Y$ containing a nontrivial path and having a compatible uniformity with a countable base which induces the open-cover topology on $Y^{x}$.

THEOREM 2. Let $X$ be a Tychonoff space, and let $Y$ be a pathwise connected and locally pathwise connected metric space. Then the following are equivalent.

1. All compatible uniformities on $Y$ induce the same topology on $Y^{x}$.

2. All compatible metrics on $Y$.induce the same topology on $Y^{X}$.

3. Either $X$ or $Y$ is pseudocompact.

For the remainder of the paper, $X$ and $Y$ will be Tychonoff spaces. The notation $M(Y)$ will be used to denote the set of all compatible uniformities on $Y$. For each $\mu \in M(Y)$, define a function from $\mu$ into the power set of $Y^{X} \times Y^{X}$ as follows. For every $U \in \mu$, let

$$
\hat{U}=\left\{(f, g) \in Y^{X} \times Y^{X} \mid(f(x), g(x)) \in U \text { for every } x \in X\right\} .
$$

It is well-known and straightforward to prove that $\{\hat{U} \mid U \in \mu\}$ is a base for a uniformity on $Y^{X}$. Let $Y_{\mu}^{X}$ denote the space $Y^{X}$ with the topology generated by this uniformity. Open sets in $Y_{\mu}^{X}$ are those sets $W$ such that for every $f \in W$, there exists a $U \in \mu$ such that $\hat{U}[f] \subseteq W$, where $\hat{U}[f]=$ $\left\{g \in Y^{X} \mid(f, g) \in \hat{U}\right\}$.

The open-cover topology on $Y^{X}$, which was mentioned above, can be defined as follows. Let $\Gamma(Y)$ denote the set of all open covers of $Y$, and for each $\mathscr{V} \in \Gamma(Y)$ and each $f \in Y^{x}$, let

$$
\begin{gathered}
\mathscr{V}(f)=\left\{g \in Y^{X} \mid \text { for every } x \in X, \text { there exists a } V \in \mathscr{V}\right. \\
\text { such that }(f(x), g(x)) \in V \times V\} .
\end{gathered}
$$


The open-cover topology is then generated by the subbase $\{\mathscr{V}(f) \mid \mathscr{V} \in \Gamma(Y)$ and $\left.f \in Y^{X}\right\}$. Denote this space by $Y_{*}^{X}$. In general, $Y_{*}^{X}$ need not be equal to $Y_{\mu}^{X}$ for any $\mu \in M(Y)$, even when $Y$ is metrizable. To see this, let $X=R$, let $Y$ be the closed unit interval in $R$, and apply Proposition 3 below.

For notational convenience, the notation $Y_{1} \leqq Y_{2}$, for topological spaces $Y_{1}$ and $Y_{2}$, will mean that $Y_{1}$ and $Y_{2}$ have the same underlying set and that the topology of $Y_{1}$ is contained in the topology of $Y_{2}$.

The open-cover topology is related to the $m$-topology which has been studied by Noble (1969). This topology has as its subbasic open sets, sets of the form $\left\{f \in Y^{x} \mid f \subseteq G\right\}$, where $G$ is a cozero subset of $X \times Y$ (here $f$ is identified with its graph). If $Y_{m}^{X}$ denotes $Y^{X}$ with the $m$-topology, if $X$ is normal and countably paracompact and if $Y$ is metrizable then $Y_{*}^{X} \leqq Y_{m}^{X}$. If in addition, $Y$ is nondiscrete, then $Y_{*}^{X}=Y_{m}^{X}$ if and only if $X$ is pseudocompact (these facts have been established by Eklund (1977)). So Theorem 1 is also true using the $\boldsymbol{m}$-topology instead of the open-cover topology whenever $X$ is normal and countably paracompact. Noble showed that if $Y$ is a nondiscrete locally compact topological group, then $Y_{\mu}^{X}=Y_{m}^{X}$ for every $\mu \in M(Y)$ if and only if $X$ is pseudocompact. It now follows from Theorem 1 that Noble's result can be extended to include all nondiscrete spaces $Y$ which are metrizable whenever $X$ is normal and countably paracompact.

When $X$ is pseudocompact and $Y$ is metrizable, then the topology of uniform convergence and the open-cover topology are the same.

Proposition 1. Let $Y$ be a metrizable space. If $X$ is pseudocompact, then $Y_{\mu}^{X}=Y_{*}^{X}$ for every $\mu \in M(Y)$.

Proof. Let $W$ be open in $Y_{\mu}^{X}$, and let $f \in W$. Then there exists a $U \in \mu$ such that $\hat{U}[f] \subseteq W$. Now for every $x \in X$, let $V_{x}$ be an open neighborhood of $f(x)$ such that $V_{x} \times V_{x} \subseteq U$. Let $\mathscr{V}=\left\{V_{x} \mid x \in X\right\} \cup\{Y \backslash f(X)\}$, which is in $\Gamma(Y)$. It is clear that $\mathscr{V}(f) \subseteq \hat{U}[f]$, so that $W$ is open in $Y_{*}^{x}$.

Let $\mathscr{V} \in \Gamma(Y)$, let $f \in Y^{x}$, and let $g \in \mathscr{V}(f)$. Since pseudocompactness is preserved by continuous functions, then $(f \times g)(X)$ is a pseudocompact subset of $Y \times Y$. Now a pseudocompact subset of a metrizable space is compact, so that $(f \times g)(X)$ is compact. Since $g \in \mathscr{V}(f)$, for every $x \in X$, there exists a $V_{x} \in \mathscr{V}$ such that $(f(x), g(x)) \in V_{x} \times V_{x}$. Also for each $x \in X$, there exists a $U_{x} \in \mu$ such that $U_{x}[g(x)] \subseteq V_{x}$. Let $U_{x}^{*} \in \mu$ such that $U_{x}^{* \circ} U_{x}^{*} \subseteq U_{x}$. Then the open cover $\left\{V_{x} \times U_{x}^{*}[g(x)] \mid x \in X\right\}$ of $(f \times g)(X)$ in $Y \times Y$ has a finite subcover $\left\{V_{x_{1}} \times U_{x_{1}}^{*}\left[g\left(x_{1}\right)\right], \cdots, V_{x_{n}} \times U_{x_{n}}^{*}\left[g\left(x_{n}\right)\right]\right\}$. Let $U=$ $U_{x_{1}}^{*} \cap \cdots \cap U_{x_{n} \cdot}^{*}$. To see that $\hat{U}[g] \subseteq \mathscr{V}(f)$, let $h \in \hat{U}[g]$ and let $x \in X$. Then there is a $k$ such that $(f(x), g(x)) \in V_{x_{k}} \times U_{x_{k}}^{*}\left[g\left(x_{k}\right)\right]$. Therefore $(g(x), h(x)) \in U \subseteq U_{x_{k}}^{*}$ and $\left(g\left(x_{k}\right), g(x)\right) \in U_{x_{k}}^{*}$, so that $\left(g\left(x_{k}\right), h(x)\right) \in$ 
$U_{x_{k}}^{*} \circ U_{x_{k}}^{*} \subseteq U_{x_{k}}$. Hence $h(x) \in U_{x_{k}}\left[g\left(x_{k}\right)\right] \subseteq V_{x_{k}}$, and thus both $h(x)$ and $f(x)$ are in $V_{x_{k}}$, which is in $\mathscr{V}$.

The metrizability of $Y$ in Proposition 1 cannot be weakened to paracompactness nor changed to compactness, as the following example illustrates. Let $[0, \Omega]$ be the ordinals less than or equal to the first uncountable ordinal with the order topology. Let $X=[0, \Omega] \backslash\{\Omega\}$, and let $Y=[0, \Omega] \times\{0,1\}$, where $\{0,1\}$ has the discrete topology. Now $X$ is pseudo-compact and $Y$ is a compact Hausdorff space and hence paracompact. Since $Y$ is compact, it has only one compatible uniformity; call it $\mu$. Let $\nu$ be the compatible uniformity on $[0, \Omega]$. For each $V \in \nu$ and for $i=0$ and 1 , let $V_{i}$ denote the set $\{((y, i),(z, i)) \in$ $Y \times Y \mid(y, z) \in V\}$. Then $\mu$ is generated by the base $\left\{V_{0} \cup V_{1} \mid V \in \nu\right\}$.

To see that $Y_{\mu}^{X} \neq Y_{*}^{X}$, first let $\xi$ be an order preserving bijection from $X$ onto the limit ordinals in $X$. For every $x \in X$, let $W_{x}=(x, \xi(x)) \times\{0,1\}$, and define $\mathscr{V}$ to be the set $\left\{W_{x} \mid x \in X\right\} \cup\{[0, \Omega] \times\{0\},[0, \Omega] \times\{1\}\}$, which is an open cover of $Y$. Also for $i=0$ and 1 , define $f_{i} \in Y^{\boldsymbol{X}}$ by $f_{i}(x)=(x, i)$ for every $x \in X$. Clearly $f_{1} \in \mathscr{V}\left(f_{0}\right)$. Let $U=V_{0} \cup V_{1}$, for $V \in \nu$, and let $g \in Y^{X}$ be such that $f_{1} \in \hat{U}[g]$. The object now will be to establish that $\hat{U}[g] \mathscr{\mathscr { V }}\left(f_{0}\right)$. Let $\pi_{0}$ and $\pi_{1}$ be the projections of $Y$ onto $[0, \Omega] \times\{0\}$ and $[0, \Omega] \times\{1\}$, respectively. Since $\pi_{0} f_{1}(X)=\varnothing$, and by the nature of $U$, it can be seen that $\pi_{0} g(X)=\varnothing$. Also since $U$ is a neighborhood of the diagonal in $Y \times Y$, there is some $x_{0} \in X$ such that $\left(\left[x_{0}, \Omega\right] \times\{1\}\right) \times\left(\left[x_{0}, \Omega\right] \times\{1\}\right) \subseteq U$.

There are two cases to consider. First suppose that for every non-limit ordinal $x$ in $X$ greater than $x_{0}, \pi_{1} g(x)<x_{0}$. Then $g \notin \mathscr{V}\left(f_{0}\right)$ for the following reason. Let $z \in X$ be such that $g\left(\xi\left(x_{0}\right)+1\right) \in W_{z}$. Since $\xi\left(x_{0}\right)+1>x_{0}$, then $\pi_{1} g\left(\xi\left(x_{0}\right)+1\right)<x_{0}$. Also $z<\pi_{1} g\left(\xi\left(x_{0}\right)+1\right)$, so that $z<x_{0}$. Since $\xi$ is order preserving, then $\xi(z)<\xi\left(x_{0}\right)$. Therefore $f_{0}\left(\xi\left(x_{0}\right)+1\right) \notin W_{z}$, so that $g\left(\xi\left(x_{0}\right)+\right.$ 1) and $f_{0}\left(\xi\left(x_{0}\right)+1\right)$ cannot both be in the same member of $\mathscr{V}$; and hence $g \notin \mathscr{V}\left(f_{0}\right)$.

On the other hand suppose there exists a non-limit ordinal $x^{\prime}$ in $X$ greater than $x_{0}$ such that $\pi_{1} g\left(x^{\prime}\right) \geqq x_{0}$. Define $h: X \rightarrow Y$ by $h(x)=g(x)$ if $x \neq x^{\prime}$, and $h\left(x^{\prime}\right)=(\Omega, 1)$. Since $x^{\prime}$ is a non-limit ordinal, then $h$ is continuous. To see that $h \in \hat{U}[g]$, note that $g\left(x^{\prime}\right) \in\left[x_{0}, \Omega\right] \times\{1\}$ and $h\left(x^{\prime}\right) \in\left[x_{0}, \Omega\right] \times\{1\}$, so that $\left(g\left(x^{\prime}\right), h\left(x^{\prime}\right)\right) \in U$. Finally, to see that $h \notin \mathscr{V}\left(f_{0}\right)$, note that $h\left(x^{\prime}\right)=$ $(\Omega, 1)$ while $f_{0}\left(x^{\prime}\right)=\left(x^{\prime}, 0\right)$; and these are not contained in the same member of $\mathscr{V}$.

Proposition 1 has the following converse involving real-valued functions. This is well-known, but its proof is included for the sake of completeness.

Proposition 2. If $R_{\mu}^{X}=R_{\nu}^{X}$ for every $\mu$ and $\nu$ in $M(R)$, then $X$ is pseudocompact. 
Proof. Suppose $X$ is not pseudocompact. Then there exists an $f \in R^{X}$ and a sequence $\left\{x_{n}\right\}$ in $X$ such that $f\left(x_{n}\right) \geqq n$ for every $n$. Let $m$ be the maximum uniformity on $R$ - that is, the neighborhoods of the diagonal (since $R$ is paracompact). For each $n$, let $U_{n}=\{(y, z) \in R \times R|| y-z \mid<1 / n\}$, and let $\mu$ be the uniformity on $R$ generated by the base $\left\{U_{n}\right\}$. Let

$$
V=\{(y, z) \in R \times R|| y-z \mid<1 /(|y|+|z|+1)\}
$$

which is an element of $m$.

To see that $\hat{V}[f]$ is not open in $R_{\mu}^{x}$, let $n$ and $g \in R^{x}$ be arbitrary. If $g \in \hat{V}[f]$, then define $h \in R^{X}$ by $h(x)=g(x)+1 / 2 n$; so that $h \in \hat{U}_{n}[g]$. Now $\left|g\left(x_{4 n}\right)-f\left(x_{4 n}\right)\right|<1 /\left(\left|g\left(x_{4 n}\right)\right|+\left|f\left(x_{4 n}\right)\right|+1\right)<1 / 4 n$, so that $f\left(x_{4 n}\right)-1 / 4 n<g\left(x_{4 n}\right)$. But then $f\left(x_{4 n}\right)+1 / 4 n<h\left(x_{4 n}\right)$, so that $\left|h\left(x_{4 n}\right)-f\left(x_{4 n}\right)\right|>1 / 4 n$. Also $1 /\left(\left|h\left(x_{4 n}\right)\right|+\left|f\left(x_{4 n}\right)\right|+1\right)<1 / 4 n$, so that $\left(h\left(x_{4 n}\right), f\left(x_{4 n}\right)\right) \notin V$. Therefore $h \notin \hat{V}[f]$, so that $\hat{U}_{n}[g] \not \hat{V}[f]$. Since $n$ and $f$ were arbitrary, then $\hat{V}[f]$ is not open in $R_{\mu}^{X}$, and thus $R_{\mu}^{X}<R_{m}^{X}$.

The full converse of Proposition 1 is true if we require $Y$ to contain a nontrivial path.

Proposition 3. Let $Y$ be a metrizable space containing a nontrivial path. If $Y_{\mu}^{X}=Y_{*}^{X}$ for every $\mu \in M(Y)$, then $X$ is pseudocompact.

Proof. Let $\rho$ be a compatible metric on $Y$, and let $\mu$ be the uniformity on $Y$ generated by the sets of the form $\{(y, z) \in Y \times Y \mid \rho(y, z)<1 / n\}$. Since $Y$ contains a nontrivial path, there is a homeomorphism $\eta$ from the interval $[0,2]$ into $Y$. Suppose that $X$ is not pseudocompact. Then there is a continuous real-valued function $\alpha$ on $X$ and a sequence $\left\{x_{n}\right\}$ in $X$ such that $\left\{\alpha\left(x_{n}\right)\right\}$ are distinct points with $\alpha\left(x_{n}\right)>n$ for each $n$. Let $\beta$ be an order preserving homeomorphism from $R$ onto the interval $(0,1)$, and let $f=$ $\eta \circ \beta \circ \alpha$. Let $y_{0}=\eta(1)$, let $y=\eta(2)$, and for each $n$, let $y_{n}=f\left(x_{n}\right)$. Choose an $\varepsilon>0$ such that $B(y, \varepsilon) \cap B(\eta([0,1]), \varepsilon)=\varnothing$, where these are the $\varepsilon$ neighborhoods of $y$ and $\eta([0,1])$, respectively. Let $\left\{V_{n}\right\}$ be a pairwise disjoint sequence of open subsets of $Y$ such that $y_{n} \in V_{n}$ and $V_{n} \cap B(y, \varepsilon)=\varnothing$ for each $n$. Let $V=Y /\left\{y_{n} \mid n=0,1, \cdots\right\}$, and define $\mathscr{V}=\left\{V_{n} \cup B(y, \varepsilon / n) \mid n=\right.$ $1,2, \cdots\} \cup\left\{V, B\left(y_{0}, \varepsilon\right)\right\}$. Now it can be seen that the constant function $c_{y} \in \mathscr{V}(f)$. Also it can be shown that if $\delta>0$ and $0<\rho(y, z)<\min \{\varepsilon, \delta\}$, then $c_{z} \notin \mathscr{V}(f)$. From this it follows that $\mathscr{V}(f)$ is not open in $Y_{\mu}^{X}$.

The hypothesis that $Y$ contains a nontrivial path in Proposition 3 cannot be omitted. To see this, consider $Q^{R}$, where $Q$ is the space of rational numbers. Then $Q_{\mu}^{R}=Q_{*}^{R}$ for every $\mu \in M(Q)$, but $R$ is not pseudocompact. 
Proposition 4. Let $Y$ be a pathwise connected and locally pathwise connected metric space. If all compatible metrics on $Y$ induce the same topology on $Y^{X}$, then either $X$ or $Y$ is pseudocompact.

Proof. Suppose that neither $X$ nor $Y$ is pseudocompact. Then there exists an unbounded continuous function $\varphi$ from $X$ into the interval $[0, \infty)$. Let $\left\{x_{n}\right\}$ be a sequence in $X$ such that $n<\varphi\left(x_{n}\right)<\varphi\left(x_{n+1}\right)$ for each $n$. Also for each $n$, let $s_{n}=\varphi\left(x_{n}\right)$; let $t_{n}, u_{n}$, and $v_{n}$ be elements of $[0, \infty)$ such that $u_{n}<s_{n}<t_{n}<v_{n}<u_{n+1}$; and let $I_{n}$ and $J_{n}$ be the closed intervals $\left[u_{n}, v_{n}\right]$ and $\left[v_{n}, u_{n+1}\right]$, respectively. Since $Y$ is not countably compact, there exists a sequence $\left\{V_{n}\right\}$ of nonempty open subsets of $Y$ which is a discrete collection in $Y$-that is, every element of $Y$ has a neighborhood intersecting at most one $V_{n}$. For each $n$, let $\psi_{n}$ be a homeomorphism from $I_{n}$ into $V_{n}$, and let $\eta_{n}$ be a homeomorphism from $J_{n}$ into $Y$ such that $\eta_{n}\left(v_{n}\right)=\psi_{n}\left(v_{n}\right)$ and $\eta_{n}\left(u_{n+1}\right)=$ $\psi_{n+1}\left(u_{n+1}\right)$. Define $f \in Y^{X}$ by $f(x)=\psi_{n} \varphi(x)$ if $x \in \varphi^{-1}\left(I_{n}\right)$, and $f(x)=\eta_{n} \varphi(x)$ if $x \in \varphi^{-1}\left(J_{n}\right)$. For each $n$, let $A_{n}=\psi_{n}\left(I_{n}\right)$, let $y_{n}=\psi_{n}\left(s_{n}\right)$, and let $z_{n}=\psi_{n}\left(t_{n}\right)$. Finally, let $A=\bigcup_{n=1}^{\infty} A_{n}$, which is a closed subset of $Y$.

Define two metrics, $\rho_{1}$ and $\rho_{2}$, on $Y$ as follows. First let $\rho$ be any given compatible metric on $Y$ which is bounded by 1 . Now let $y, z \in A$; say $y \in A_{m}$ and $z \in A_{n}$. If $m \neq n$, then take $\rho_{1}(y, z)=1$ and $\rho_{2}(y, z)=1$. If $m=n$, define $\rho_{1}(y, z)=(1 / n) \rho(y, z)$ and $\rho_{2}(y, z)=\min \left\{1, \rho(y, z) / \rho\left(y_{n}, z_{n}\right)\right\}$. Then $\rho_{1}$ and $\rho_{2}$ are metrics on $A$ compatible with the subspace topology on $A$. Since $A$ is closed in $Y, \rho_{1}$ and $\rho_{2}$ can be extended to compatible metrics on $Y$ (see for example Bing (1947)).

To see that $B_{\rho_{1}}(f, \delta)$ is not contained in $B_{\rho_{2}}(f, 1)$ for any $\delta$, let $\delta>0$ be arbitrary. Choose integer $n$ greater than $1 / \delta$. Now let $\alpha$ be a homeomorphism from $I_{n}$ into $I_{n}$ which takes $s_{n}$ to $t_{n}$ and is fixed on $u_{n}$ and $v_{n}$. Define $g \in Y^{x}$ by $g(x)=f(x)$ if $x \in \varphi^{-1}\left(I_{m}\right)$ for $m \neq n$, and $g(x)=\psi_{n} \alpha \varphi(x)$ if $x \in \varphi^{-1}\left(I_{n}\right)$. Since $f$ and $g$ differ only on $A_{n}$, and since the diameter of $A_{n}$ with respect to $\rho_{1}$ is less than $1 / n$, then $g \in B_{\rho_{1}}(f, \delta)$. However, $f\left(x_{n}\right)=y_{n}$ and $g\left(x_{n}\right)=z_{n}$, while $\rho_{2}\left(y_{n}, z_{n}\right)=1$. Therefore, $g \notin B_{\rho_{2}}(f, 1)$, so that $B_{\rho_{1}}(f, \delta) \notin B_{\rho_{2}}(f, 1)$. From this it follows that the topologies on $Y^{X}$ induced by $\rho_{1}$ and $\rho_{2}$ are different.

\section{References}

R. H. Bing (1947), 'Extending a metric', Duke Math. J. 14, 511-525.

A. Eklund (1977), Dissertation, Virginia Polytechnic Institute and State University.

A. Irudayanathan (1967), Dissertation, Iowa State University.

R. A. McCoy (to appear), 'The open-cover topology on function spaces', Fund. Math.

N. Noble (1969), 'Products with closed projections', Trans. Amer. Math. Soc. 140, 381-391.

H. Poppe (1966), 'Ein Kompaktheitskriterium für Abbildungsraume mit einer verallgemeinerten uniform Struktur', Second Prague Conference in Topology.

Virginia Polytechnic Institute and State University,

Blacksburg, Virginia 24061. 